\title{
La Historia Moderna en la Enseñanza Secundaria
}

Contenidos, métodos y representaciones

Francisco García González, Cosme J. Gómez Carrasco Ramón Cózar Gutiérrez, Pedro Martínez Gómez (Coords.)

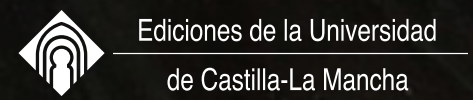





\section{LA HISTORIA MODERNA EN LA ENSEÑANZA SECUNDARIA. CONTENIDOS, MÉTODOS Y REPRESENTACIONES}





\section{LA HISTORIA MODERNA EN LA ENSEÑANZA SECUNDARIA. CONTENIDOS, MÉTODOS Y REPRESENTACIONES}

Francisco García González

Cosme J. Gómez Carrasco

Ramón Cózar Gutiérrez

Pedro Martínez Gómez

(coords.)

Ediciones de la Universidad

Cuenca, 2020 
(C) de los textos: sus autores

(c) de la edición: Universidad de Castilla-La Mancha

Edita: Ediciones de la Universidad de Castilla-La Mancha

\section{Colección JORNADAS Y CONGRESOS n. ${ }^{\circ} 27$}

Imagen de cubierta: Felipe V, Rey de España (1624). Diego Velázquez. (CC0 1.0) Metropolitan Museum

El procedimiento de selección de originales se ajusta a los criterios específicos del campo 10 de la CNEAI para los sexenios de investigación, en el que se indica que la admisión de los trabajos publicados en las actas de congresos deben responder a criterios de calidad equiparables a los exigidos para las revistas científicas y capítulos de libros.

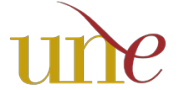

Usión de EprToriales

Esta editorial es miembro de la UNE, lo que garantiza la difusión y comercialización de sus publicaciones a nivel nacional e internacional

ISBN: 978-84-9044-400-9

D.O.I.: http://doi.org/10.18239/jornadas_2020.27.00

Composición: Compobell

Hecho en España (U.E.) - Made in Spain (U.E.)

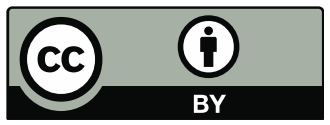

Esta obra se encuentra bajo una licencia internacional Creative Commons CC BY 4.0.

Cualquier forma de reproducción, distribución, comunicación pública o transformación de esta obra no incluida en la licencia Cretative Commons CC BY 4.0 solo puede ser realizada con la autorización expresa de los titulares, salvo excepción prevista por la ley. Puede Vd. acceder al texto completo de la licencia en este enlace: https://creativecommons.org/licenses/by/4.0/deed.es
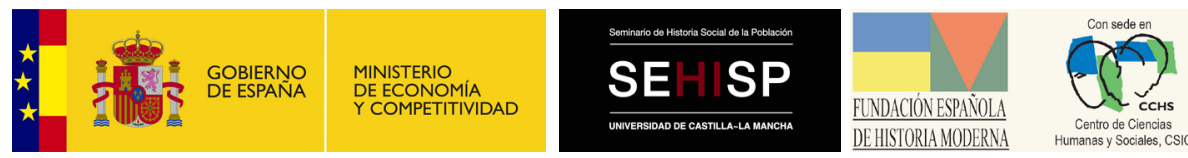


\section{ÍNDICE}

INTRODUCCIÓN............................

Francisco Garcia González, Cosme Jesús Gómez Carrasco, Ramón

Cózar Gutiérrez y Pedro Martinez Gómez

\section{LOS CONTENIDOS SOBRE LA EDAD MODERNA EN EDUCACIÓN SECUNDARIA}

A época moderna nos manuais escolares portugueses: um balanço entre história regulada, história ensinada e história desejada ....... Cristina Maia

Análisis del currículum de Historia Moderna en Enseñanza Secundaria desde la pedagogía crítica $\ldots \ldots \ldots \ldots \ldots \ldots \ldots \ldots$ Sofía Díaz de Greñu Domingo

Contrastes de la presencia de la Edad Moderna en los libros de texto

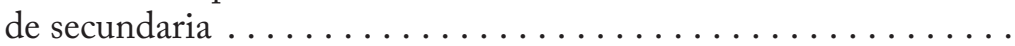

Gemma Muñoz García, Ma Montserrat Pastor Blázquez y José

Manuel Gómez Contreras

El papel de la mujer en la enseñanza de la Historia Moderna....... . Antuanett Garibeh Louze y Rafael Duro Garrido

Imágenes e ilustraciones de la Edad Moderna en los manuales de ESO (de la LOGSE a la LOMCE)..................... Raimundo A. Rodríguez Pérez

La enseñanza de la Historia Moderna en los manuales escolares en el tránsito de la Educación Primaria a la Secundaria a partir de hitos significativos: América, el Imperio español y la Guerra de Sucesión Juan Manuel Casanova García

La idea de decadencia durante el reinado de los Austrias en el currículum de la LOMCE: la vigencia del paradigma decimonónico .... Francisco Rubino 
Las minorías ibéricas de la Edad Moderna. Moriscos y judeoconversos en los libros de texto de Enseñanza Secundaria (1970-2010). Una

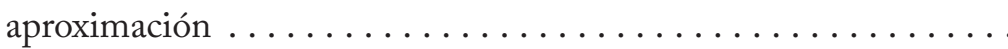
Francisco J. Moreno Díaz del Campo

Tiempo histórico, longuée durée y sistema-mundo en la Enseñanza Secundaria: una didáctica de las teorías de Wallerstein y Braudel . Jorge Velasco Baleriola

El conocimiento de la historia local a través de los principios científico-didácticos: la conquista de Illora y su organización señorial . . . José Antonio Jiménez López

El reino de Granada tras la incorporación a Castilla en los manuales de Bachillerato andaluces . . . . . . . . . . . . . . . . . . Valeriano Sánchez Ramos

El uso de la historia local en la enseñanza de la Historia Moderna. El proyecto "Alcalá en el aula"........................ Javier Jiménez Rodríguez

Relegadas a un segundo plano: la figura femenina en los currículos de

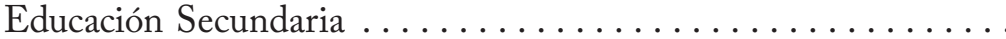
Begoña Martinez San Nicolás

Historia del arte con perspectiva de género. Diseño de materiales de Secundaria y Bachillerato . . . . . . . . . . . . . . . . . . Elvira Sanjuán Sanjuán

Análisis sobre el estudio de la Historia Moderna en un instituto de Enseñanza Secundaria en territorio cervantino ............. Juan de Á. Gijón Granados

\section{ENSEÑAR LA EDAD MODERNA EN EDUCACIÓN SECUNDARIA}

La Historia del Arte de época moderna no es aburrida: la creatividad como herramienta didáctica en la universidad . . . . . . . . . . . Aintzane Erkizia-Martikorena

Aprender la Historia Moderna desde las fuentes en Educación Secundaria ................................

Victor Pampliega Pedreira y Alba de la Cruz Redondo

Innovación y prácticas de aula a través de dos propuestas: administrar la Monarquía hispánica y viajar por la España ilustrada........ Juan Diaz Álvarez, Carla Rubiera Cancelas, Fernando Rodríguez del

Cueto, Maria Álvarez Fernández y Jorge Muñiz Sánchez 
El callejero de La Carolina (Jaén) como fuente de conocimiento de la

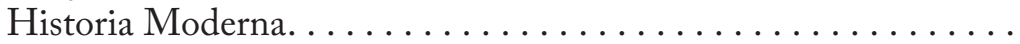

¿Qué preguntas? ¿Qué problemas? La enseñanza “crítica” de la Historia Moderna . . . . . . . . . . . . . . . . . . . . . . . . . . . Marta Marín Sánchez

Tecnologías emergentes al servicio de la evaluación en la enseñanza de la Historia Moderna. . . . . . . . . . . . . . . . . . . . . Ramón Cózar Gutiérrez y Francisco de Borja Caparrós Ruipérez

Repoblando el futuro en las tierras de Albacete. Proyecto de innovación basado en el aprendizaje-servicio. IES Amparo Sanz de Albacete. Cursos 2016-2018 . . . . . . . . . . . . . . . . . . . . . . . Carmen Hernández López y Carmen Monzó González

"Cuartos de maravillas y gabinetes de curiosidades". Una propuesta de Aprendizaje Basado en Proyectos en torno a la ciencia y la cultura

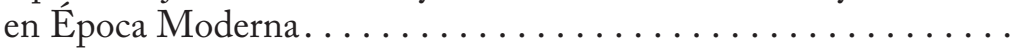
Lorena Álvarez Delgado y Ana Luisa Martinez Carrillo

Ensenada en el Bachillerato: los cultivos en el reino de Córdoba a mediados del Setecientos . . . . . . . . . . . . . . . . . . . . . $M^{a}$ Soledad Gómez Navarro y Eduardo Lama Romero

El enemigo invisible: la peste. Una propuesta de estudio de la epidemia de Lucena de 1679 a través de las nuevas tecnologías. . . . . . . . . . Elena X. Paoletti Ávila

Entre llaves y documentos: el uso didáctico de un escape room sobre la Edad Moderna en la formación inicial del profesorado ......... Mercedes de la Calle Carracedo, María Sánchez-Agusti, Esther López

Torres, José M. ${ }^{a}$ Martinez Ferreira, Diego Miguel-Revilla, y M. ${ }^{a}$

Teresa Carril Merino

Flipped classroom, gamificación y evaluación continua en la docencia de la historia precolombina y colonial de América ............ Antonio Carrasco Rodríguez

Hacia una didáctica del Madrid de los Austrias: una propuesta desde el plano de Teixeira (1656) . . . . . . . . . . . . . . . . .

David Alonso García

La cultura morisca: nuevos espacios y métodos de aprendizaje para el

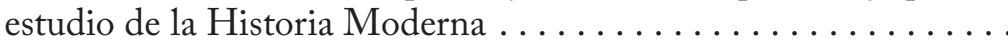
Julia Hernández Salmerón 
Metodología y propuesta didáctica sobre la población de Cáceres en 1787a través de las TIC's . . . . . . . . . . . . . . . . . . . . .

Raquel Tovar Pulido

La Edad Moderna a debate: la oratoria y la discusión como método

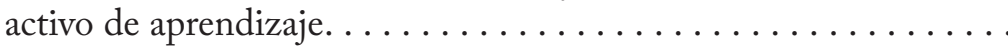
Milagros León Vegas

Historia de la música durante los siglos XVI-Xviı. Una propuesta

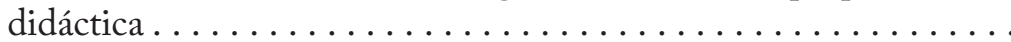
Ricardo Largo Martín

Imágenes del Descubrimiento de América en los libros de texto de España y México . . . . . . . . . . . . . . . . . . . . . . . . . Ana I. Irigoyen Bueno

La novela de los siglos XVI y XVII como fuente primaria para el conocimiento de la Edad Moderna. . . . . . . . . . . . . . . . . . . . . José Andrés Prieto Prieto

La obra de Vandelvira en la provincia de Albacete (propuesta didáctica $3^{\circ} \mathrm{ESO}$ y $2^{\circ}$ de Bachillerato) . . . . . . . . . . . . . . . . . . . $M^{a}$ del Mar Rodríguez Ruiz

Las dinámicas de rol en la gamificación de la enseñanza de la Historia

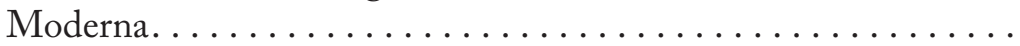
Antonio Carrasco Rodríguez

Las nuevas poblaciones de Sierra Morena y Andalucía en la España de Carlos III: una propuesta didáctica a partir de la legislación y la novela histórica. ............................ Adolfo Hamer-Flores

Los itinerarios calatravos. Una propuesta didáctica a través de las órdenes militares de Castilla . . . . . . . . . . . . . . . . . . . . Héctor Linares González

Mujeres en la historia. El proyecto "Identidad e imagen de Andalucía en la Edad Moderna" como recurso didáctico. . . . . . . . . . . . . . Maria del Mar Felices de la Fuente, Domingo Marcos Giménez Carrillo y Francisco Gil Martinez

Revueltas y revoluciones en la Edad Moderna. Una oportunidad didáctica ................................ Joaquim Enric López Camps 
Una propuesta de recursos digitales interactivos para la enseñanza y el aprendizaje de los avances científicos y tecnológicos de la Historia Moderna.................................. Ana Luisa Martinez Carrillo y Lorena Álvarez Delgado

Conectando el pasado. La enseñanza de la historia en Bachillerato a

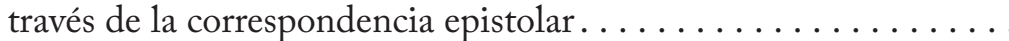
Alvaro Chaparro Sainz y Rafael Guerrero Elecalde

Una forma del uso del ABP y del flipped classroom en la enseñanza de la Historia Moderna a través de la novela picaresca ........... Pedro Antonio Amores Bonilla

Posibilidades concretas de desarrollo de contenidos de Historia

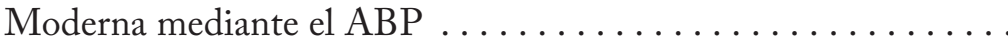
Pedro Antonio Amores Bonilla

Una mirada a la enseñanza en Lorca durante la Edad Moderna. Del

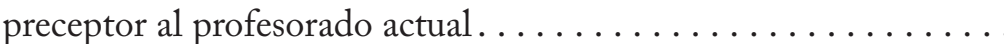
Rafael Gil Bautista y Carmen Gil Huedo

Una propuesta metodológica para el estudio del gremio en el aula. Las trayectorias de los linajes artesanos durante la Edad Moderna.... Francisco Hidalgo Fernández

Sacar la Edad Moderna a la calle: la Marchena de Diego López de Arenas $(1576-1640)$. . . . . . . . . . . . . . . . . . . . . . Francisco Javier Gutiérrez Núñez

El estudio de la Corte y el estilo de vida cortesano en la Educación

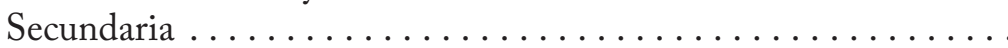
David Quiles Albero

\section{LA IMAGEN DE LA EDAD MODERNA EN EL TIEMPO Y EN LA SOCIEDAD ACTUAL}

"Vivir como un canónigo". Estereotipos y realidades de los componentes de una élite social y económica del Antiguo Régimen ....... Santos Jaime Valor

Recursos didácticos con piezas del Museo de América que acercan a la ESO otras realidades de Época Moderna.................. 603 Gemma Ma Muñoz Garcia y Esther Jiménez Pablo 
Protegiendo las murallas. La adopción del patrimonio de época moderna desde la Educación Secundaria ................ Antoni Bardavio Novi y Sònia Mañé Orozco

Del acto a la identidad: la utilidad del estudio de la diversidad sexual

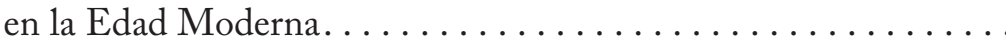
Juan Pedro Navarro Martinez

Desmontando la Leyenda Negra ..................... José Andrés Prieto Prieto

Didáctica crítica de la historia a través del cine: la «cultura popular〉 en la Edad Moderna . . . . . . . . . . . . . . . . . . . . . . . Gustavo Hernández Sánchez

$\mathrm{El}$ cine como fuente de representaciones sociales de "El descubrimiento y conquista de América" .......................... Andrea M. Ordónez Cuevas y Nicolás Pozo Serrano

El descubrimiento y la conquista de América en la televisión de la transición española: la censura del episodio "E1 Siglo de Oro español" de la serie Érase una vez... El hombre .................. Julián Pelegrín Campo

El siglo XviI a través del arte Barroco. El trabajo con cuadros vivientes como aplicación en el aula ...................... Raúl Alcabut Utiel

Mujeres olvidadas en la historia. Estereotipos e invisibilidad en los libros de texto . . . . . . . . . . . . . . . . . . . . . . . . Daniel Maldonado Cid

Enseñando Historia Moderna en las aulas: didáctica con videojuegos Teresa Cantó Gomis

Lexical choices in the characterisation of King Henry vin in the TV

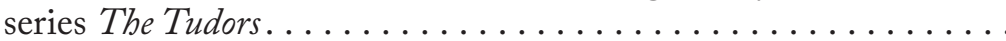
Verónica Falquet Aparisi

Las variaciones del clima a través de la "Gran historia": algunas consideraciones para la enseñanza de la Edad Moderna........... . Francisco-Javier Rubio-Muñoz, Alejandro Gómez-Gonçalves y Diego Corrochano-Fernández

La imagen de la Edad Moderna a través de los videojuegos de temáti-

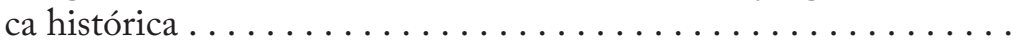
Maria de la Encarnación Cambil-Hernández, Daniel Camuñas-García y Rafael Marfil-Carmona 
La imagen del cardenal Francisco Jiménez de Cisneros 500 años después 753 Omar Gómez-Cornejo Aguado

La Guerra de Secesión española en la Educación Secundaria. Contenidos, materiales didácticos y propuesta metodológica ........ 761 Victor Alberto García Heras

Individualismo. Discursos, prácticas y estereotipos en la Edad Moderna 771 Carlos Vega Gómez

La paleografía como recurso didáctico interdisciplinar en el aula de Secundaria ................................ 781

David Martín López y Francisco Fernández Izquierdo

Los mass-media como generador de conocimiento ............ 793

Isabel Escalera Fernández

Los sitios reales como espacios para la enseñanza. Historia, patrimonio

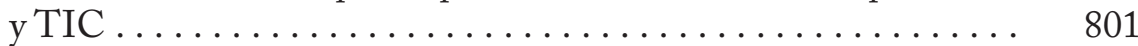

Jorge Pajarin Dominguez

Nobleza y oligarquía en femenino. Un ejemplo de ascenso social en la ciudad de Granada durante el Antiguo Régimen............ Javier García Benitez

Una visión novedosa de Gonzalo Fernández de Córdoba desde la

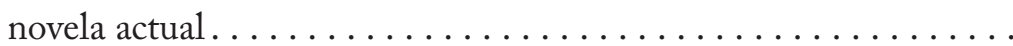
Andrés Palma Valenzuela

La imagen del poder. El poder de la imagen en la Edad Moderna ... 837 Rafael Gil Bautista y Carmen Gil Huedo 



\title{
LOS MASS-MEDIA COMO GENERADOR DE CONOCIMIENTO
}

\author{
ISABEL ESCALERA FERNÁNDEZ \\ (Universidad de Valladolid) \\ http://doi.org/10.18239/jornadas_2020.27.67
}

\section{INTRODUCCIÓN}

Las posibilidades que ofrece el mundo actual potencian el surgimiento de nuevos métodos que incorporar en la enseñanza, la cual se ve enriquecida con los análisis que se plantean desde distintas perspectivas. La aparición de los mass media ha tenido como consecuencia una ampliación del espectro, haciendo posible que los diversos individuos que se encuentran en la sociedad sean capaces de comunicarse. En este sentido, Julio Cabero (1996) sostuvo que la era en la que estábamos inmersos había cambiado totalmente, puesto que la tecnología no solo beneficia a la comunicación, sino que modifica el paradigma en el que nos hallábamos inmersos. Como consecuencia de esta transformación, el mercado tuvo que adaptarse a marchas forzadas para satisfacer la demanda. Así Marín (2012) afirmó que se incorporaron con gran celeridad al carro del avance.

Además, los medios de comunicación juegan un papel relevante en nuestra sociedad. García (2003) mencionó que eran los intercesores entre lo que pasaba y el público y, por su puesto, eran referentes en la industria cultural. Esta visión fue seguida en parte por Méndez (2001), quien reconoció el peso que tenían los mass-media en la cotidianidad debido a que cumplen una doble función. Por un lado, atestiguan lo que acontece en la sociedad y, por otro lado, entretienen. Así pues, hablar de espacio-tiempo resulta inexacto, ya que:

Los medios de comunicación desempeñan un papel central en la dinámica de las sociedades actuales más desarrolladas:a) como mediadores entrelos acontecimientos y el público, b) como referentes del poder, c) como industria cultural, d) 
como empresas básicas de la nueva economía, e) como agentes de la democracia, f) como portavoces de intereses ciudadanos (García Galindo, 2003).

El propio Becerril (2005) subrayó que "Si la tecnología es el resultado de una cultura, en ella se están transmitiendo los valores, normas y comportamientos propios". Si los mass media tienen tanta importancia en nuestra sociedad, lo lógico es pensar ¿cuáles son los que más incidencia poseen? Pues bien, la Asociación Española de Videojuegos (AEVI) publicó en sus resultados anuales que tanto internet como los videojuegos son los más importantes. Así, las cifras que se alcanzaron en 2014 vendiendo videojuegos, bien fuese en venta física o en venta online, eran un claro ejemplo del lugar preeminente que desempeñaban en la sociedad.

Corominas (1994) señaló que "tienen un papel de transmisión de conocimiento que básicamente se da por vías emotivas, impactantes y de identificación con el espectador". No obstante, han sido objeto de numerosas controversias y se ha puesto en duda su papel como recurso didáctico. Sin embargo, decir que los videojuegos solo cumplen una función meramente lúdica es un error, puesto que en el área de la educación sus posibilidades son infinitas.

En este caso los videojuegos no son solo un mero pasatiempo, sino que pueden ser una herramienta fundamental para estudiar, al igual que sucede con las series. El hecho de querer innovar no es baladí,ya que el aprendizaje se encuentra fuertemente vinculado a la acción transformadora del mundo. Por tanto,el sentido que se confiere a la educación es fundamental al producir unas características que nunca se podrían conseguir de manera espontánea.Así,estas deben ser cuidadosamente organizadas para que, a la hora de llevar a cabo esta innovación o aprendizaje, los efectos que se produzcan beneficien a todo el conjunto de la sociedad.

Antes de implementar esta nueva medida, se debe reflexionar sobre la repercusión que puede tener en la mejora del estudio, al fin y al cabo, el propósito principal es contribuir al mejor entendimiento de los materiales. Resulta insoslayable analizar si estos cambios son meramente un beneficio a corto plazo o si realmente se puede sacar provecho de su uso.En resumen,se trata de comprender si lo que se estállevando a cabo es útil y puede llevar consigo una transformación en la mejora educativa, puesto que, como sostiene Jiménez (2018): "el videojuego es una producción esencialmente destinada al ocio resultado de la aplicación de las tecnologías digitales".

\section{PROPUESTA DIDÁCTICA}

En esta propuesta dos van a ser los mass-media empleados: las series y los videojuegos. Si bien es cierto que las series de televisión han sido más estudiadas desde distintos ámbitos, no lo han sido tanto los videojuegos. Con el objetivo 
de procurar una mejor educación y aprovechar los recursos que nos ofrecen los videojuegos se ha creado lo que se conoce como gamificación. Esta metodología posibilita aunar la historia que contenga el videojuego y aplicarla en el aula como soporte a la lección. Uno de los factores que destaca sobremanera en ellos es su elevado contenido visual, haciendo que los estudiantes puedan aprender $\mathrm{y}$, a su vez, asentar la información recibida.

Frente a otras metodologías más tradicionales, la gamificación está logrando una amplia repercusión gracias al carácter lúdico que la envuelve. Por lo tanto, hay una experiencia positiva, la cual es, sin duda, el aprendizaje de los contenidos por parte del alumnado. El hecho de elegir las series y los videojuegos plantea un enfoque diverso puesto que la manera en la que ambas se relacionan con el espectador es diametralmente distinta. Así, mientras que en las series el espectador es pasivo, en los videojuegos la persona que está jugando puede elegir qué hacer y dónde ir. En definitiva, es un sujeto plenamente activo. Marín (2012) mencionó esto cuando afirmó que "su gran acción audiovisual, su dinamismo [...], son estos los principales elementos que los hacen tan atractivos para el público al que están destinados".

La propuesta didáctica de este estudio consiste en analizar las escenas que se presentan tanto en el videojuego Assassin's Creed como en la serie Da Vinci's Demons y aplicarlas para entender mejor los contenidos de las asignaturas. En este caso el ejemplo que se pone tiene que ver con asignaturas que versen sobrela Edad Moderna, concretamente en un momento y lugar determinados, el renaci- miento italiano.La serie de televisión DaVincis Demons fue estrenada en el año 2013, siendo distribuida internacionalmente. Esta contó con tres temporadas, finalizando su emisión en 2015. Narra la vida del artista Leonardo da Vinci, así como la de los personajes para los que trabajó, como son los Médici.

Por otra parte,el videojuego Assassin's Creed recrea fielmente el Renacimiento en Italia.Esta saga es bien conocida por el detalle que se puso a la hora de recrear

los escenarios. De hecho, para su desarrollo, tuvieron que contar con la ayuda de expertos en estas materias para poder llevarlo a cabo.En este juego puedes pasear por Florencia y encontrarte a Maquiavelo, entras en el taller de Leonardo da Vinci,trepas por la Capilla Sixtina,observas la construcción dela cúpula por parte de Brunelleschi,etc.En definitiva, a través de todos estos escenarios,el estudiante puede comprender mejor las diferentes épocas históricas.

\section{APRENDER DISFRUTANDO}

Los videojuegos no son vistos por toda la sociedad como algo que sirva para aumentar la erudición de los jóvenes estudiantes. Es más, generalmente lo prime- 
ro que se suele decir al hablar de ellos es el alto grado de violencia que contienen sus imágenes (Marín y García, 2003). No podemos negar el hecho de que existen videojuegos y series con esta temática agresiva, pero sí que merece la pena señalar que en el estudio que realizó la AEVI los videojuegos más vendidos en el país eran los que tenían como temática la aventura gráfica y todos aquellos relacionados con el baile. Calvo (1995) señaló que los videojuegos poseen la capacidad de insertarse en distintos campos de la sociedad y, desde luego, la educación es uno de ellos.

De este modo, tanto los videojuegos como las series pueden incorporarse al ámbito educativo. En lo relativo a los primeros destaca Assassin's Creed, el cual forma parte de una saga que aborda las cruzadas. No obstante, la segunda parte es la que nos interesa porque versa sobre Italia, en concreto la Italia renacentista. Su protagonista se llama Ezio Auditore, cuya familia sufre una traición a manos de las familias que supuestamente eran aliadas. Cuando el joven va a visitar a su padre, este le revela un lugar secreto donde encontrar distintos objetos que le permitirán vengarse.

Uno de los aspectos que más destaca en el videojuego es el interés que se puso por parte de los creadores para recrear de manera minuciosa la Italia renacentista. Además, el personaje se mueve por distintos lugares del país, por lo que le vemos en Monteriggioni, una ciudad de la Toscana, así como por Florencia, Venecia, etc. Eso nos permite observar no solo el plano de las ciudades, sino también los edificios más importantes del momento como el Palacio Pitti (Escalera, 2019). Asimismo, podemos asistir a alguno de los acontecimientos históricos más relevantes, como el enfrentamiento de la familia Pazzi y la familia Médici. No se debe olvidar que la propia historia de Ezio gira en torno a la traición entre familias, de todo ello se deduce como corolario que es un aspecto fundamental para entender la Italia del momento. Sin embargo, dentro del propio videojuego podemos rastrear otro discurso y es que:

Entre las críticas que el juego hace a la sociedad actual, hay un tema que resalta y es uno de los núcleos temáticos más importantes de la narración: la historia oficial, la historia tal como nos es transmitida, no es simplemente la narración de los hechos tal como sucedieron, sino la versión que los vencedores nos han legado y en la que los vencidos han sido silenciados (Martín,2012).

En lo concerniente a la serie Da Vinci's Demons, la trama gira alrededor de este icónico personaje. La primera temporada de la serie se sitúa en Florencia en el siglo XV.De este modo, tanto la serie como el videojuego están analizando un mismo periodo histórico, dado que Ezio coincide con el propio artista durante el transcurso del videojuego. 
Sin duda,una de las cosas que más fascinación ha causado fueron los inventos que llevó a cabo. Así se afirma:

Al observar sus bocetos nadie puede evitar sentirse doblemente subyugado, tanto porlaindiscutiblebellezade susdibujoshechoscondelicadosyprecisos trazos desanguinay carbonilla,comopor la minuciosaydetalladadescripción de los mecanismos y de las herramientas empleados en sus proyectos.En los esbozos de Leonardo, hasta los engranajes son bellos (Tomasini,2012).

A través de la serie se pueden rastrear los distintos inventos que concibió,como los artefactos que tenían por objeto ser armamento de guerra,como las catapultas. Asimismo,destaca sobremanera su artilugio volador,el cual no solo aparece en la serie, sino que también es uno de los elementos que Ezio emplea.

Con respecto a los sucesos históricos, a lo largo de los capítulos el espectador es consciente de la rivalidad que existe entre las dos familias: los Médici y los Pazzi. Esto también va a ser un elemento de unión entre ambos mass-media, sin embargo, hay una serie de diferencias. Mientras que en el juego el conflicto se desarrolla de manera somera,en la serie son varios capítulos los que atestiguan el enfrentamiento, culminando uno de ellos con la huida de Lorenzo y la muerte de Giuliano.

\section{DISCUSIÓN Y CONCLUSIONES}

Los videojuegos, al ser tan recientes, resultan a priori más complicados de emplear en la educación que las series.Empero, el cambio en la propuesta didáctica lleva consigo un análisis pormenorizado de los contextos en los que este tipo de recursos se pueden usar (Escalera y García, 2018). Gros y Bernat (2008) sostienen que "el diseño de los contextos de aprendizaje se convierte en una de las tareas básicas para el profesor, por lo que el rol de este cambia de forma muy notable”.También se debe tener en cuenta que no es lo mismo enfrentarse a un videojuego fuera delámbito educativo que en un ambiente formal y creado específicamente paraello.

En este caso, los dos videojuegos que se han analizado se insertan en una diná- mica que resulta favorable para aprender Historia e Historia del Arte. Esto no quiere decir que el resto de las disciplinas queden relegadas a un segundo plano, sino todo lo contrario. Así, a través de ambas propuestas uno puede estudiar el urbanismo de las distintas ciudades italianas, la vestimenta de los personajes, las armas, los procesos arquitectónicos del momento, el arte y los inventos de Leonardo. En definitiva, ambos aportan una cantidad de información que resulta totalmente favorable a la hora de profundizar en cualquiera de estos campos. De este modo: 
[...] no dejaría de ser una cuestión tangencial si detrás de muchos de los videojuegos no pudiésemos hallar profundos contenidos de carácter humanístico. No solo se trata de la narrativa que subyace en el desarrollo de sus guiones, en la iconografía que se ve, con recursos de imagen que cuentan con la complicidad cultural del usuario [...] (Jiménez, 2018).

Con ellas el alumno puede aprender sin la necesidad de estar haciendo un gran esfuerzo.Hace años se negaba que el cine pudiese servir para estudiar y se rechazaba en gran medida en el aula. Hoy en día, muchas de las dinámicas que se hacen con los estudiantes tienen que ver con el análisis de los contenidos de las películas.Así que ¿jpor qué rechazar los mass-media cuando estos pueden servir como material adicional para el aprendizaje? Quizá después de haber superado hace unos años la asignatura, el alumno no se acordará de en qué año se construyó la cúpula de la catedral de Florencia, pero es probable que sí que recuerde la recreación de la misma gracias a los medios de masas, en este caso los videojuegos y las series.

\section{REFERENCIAS BIBLIOGRÁFICAS}

Calvo,A.M.(1995).Videojuegos:deljuegoalmediodidáctico.Comunicaciónypedagogía: Nuevas tecnologías y recursos didácticos, 152,63-70.

Corominas,A.(1994).Lacomunicación audiovisual ysu integración en el currículo. Barcelona: Graó.

Escalera, I. y García, B. (2018). Aprender historia a través del píxel. En 3rd Virtual International Conference on Education, Innovation and ICT (pp.11-14). Eindhoven: Adaya Press.

Escalera, I. (2019). Gamificación en la educación: el rol de la Historia del Arte. En 3rd International Virtual Conference on Educational Research and Innovation (pp. 65-69). Madrid: REDINE.

García Galindo,J.A.(2003).La universidad y los medios de comunicación al servicio de la sociedad.EnJ.I.Aguaded(Coord.),Lucesenellaberintoaudiovisual.Librodeactas (pp.167170).Huelva: Universidad de Huelva.

Hidalgo Vásquez,X.(2012).Videojuegos, unarteparala historiadelarte(Tesis doctoral).

Universidad de Granada, Granada.

Jiménez Alcázar,J. F.(2016). De la Edad de los Imperios a la Guerra total: Medievo y videojuegos. Murcia: Compobell.

Jiménez Alcázar, J. F. (2018). La historia vista a través de los videojuegos. En P. Suárez, M. ${ }^{a}$ I. Gascón, L. Alonso y J.F. Jiménez (Coords.), Juego y ocio en la historia. (pp. 141170). Valladolid: Universidad de Valladolid. 
Lacasa,P.(2011).Los videojuegos.Aprenderen mundosrealesy virtuales.Madrid:Ediciones Morata. Marín Díaz,V.(2012).Los videojuegos ylosjuegos digitales como materiales educativos.

Madrid: Síntesis.

Marín,V.y García,M.D.(2003).La familia e internet, ¿un juego a tres bandas? Comunicar: Revistacientificaiberoamericanadecomunicaciónyeducación,21,123-126.

Martín, G. (2012). Estética de la creación virtual. Aproximación a los videojuegos en tanto que discursos culturales: el caso de Assassin's Creed. Question, 36, 16-24.

Meijer,C.J.W.(2003).Educación Inclusiva y Prácticas en el aula.Bruselas: Agencia Europea para el Desarrollo de la Educación Especial.Recuperado de https://www.european- agency.org/ sites/default/files/special-needs-education-in-europe_sne_europe_es.pdf

Méndez,J.M.(2001).Aprendemosa consumirmensajes.Televisión,publicidad,prensa, radio.

Huelva: Comunicar Ediciones.

Rosas R., Nussbaum,M., Cumsille, P.,Marianov, V.,Correa,M.,Flores, P...,Salinas, M. (2003). Beyond Nintendo: design and assessment of Educational videogames for first and second grade students. Computers E Education, 40,71-94.

Tomasini,M.C.(2012).Las máquinas de Leonardo da Vinci.Cienciay Tecnología, 12, 27-36. 
